\title{
Cortical deafness as a sequela to meningitis: a single case study
}

\author{
Megha Sasidharan, Jinsi Elsa Itty, Ghanta Hinduja, Shabnam Hasna, Deena Priya*
}

Dr. S.R. Chandrasekhar Institute of Speech and Hearing, Bengaluru, Karnataka, India.

\begin{abstract}
SUMMARY The study reports a case that was responding well to sounds and suddenly stopped hearing following fever. She contracted bacterial meningitis at the age of 5 months and had sought an audiological opinion at the age of 7 years. On evaluation, the objective test results showed normal peripheral hearing, but behaviorally she did not respond to any sounds presented during pure-tone audiometry (PTA). Thus, she was evaluated for higher auditory function using late latency response (LLR) and the responses were absent bilaterally. This confirmed cortical deafness post meningitis. Meningitis can thus also cause cortical deafness in addition to peripheral hearing loss.
\end{abstract}

Keywords meningitis, pure-tone audiometry, late latency response

\section{Introduction}

Cortical deafness is a condition where a patient presents with normal peripheral hearing but may be unable to hear the sounds due to a lesion in the auditory cortex. The literature primarily shows evidence of cortical deafness only through limited case studies (1-4). In addition, the available data is not considered due to lack of consensus in the nomenclature (5) and lack of a consistent protocol in the assessment of cortical deafness (6). Thus, understanding of this disorder is partial.

Some of the causes for cortical deafness reported in the literature reveal head injury/trauma, cerebro vascular accident (CVA), infections $(3,7,8)$ and seldom meningitis (9). The literature on meningitis focuses primarily from the view of a peripheral lesion, which became more evident with the advent of cochlear implants. In addition, ossification is a primary consequence of meningitis. It starts as early as 21 days after infection and is completed within a few months usually less than six months (10). Ossification in meningitis has been deeply studied to evaluate the benefits from cochlear implants (11).

\section{Case Report}

The case presented in this study is a 7-year-old girl who was first seen for a hearing screening at a village camp. She presented with not hearing nor speaking age appropriately and was referred for a detailed audiological evaluation. Her history and medical records revealed that she was admitted for evaluation of a high-grade fever at the age of 5 months, was diagnosed as acute bacterial meningitis, and was treated accordingly. A computed tomography (CT) scan finding was normal and no other imaging reports were available. A neurosonogram was recommended but a follow up was not done. The parents reported that until the age of 5 months the child used to respond to sounds and suddenly stopped responding, following the onset of fever.

At the age of 7 years when she reported at our institute for the purpose of receiving a handicap certificate, she did not follow verbal instructions but could understand gestural commands. She was conditioned to respond to pure-tone audiometry (PTA) by making her raise her hand on the presentation of a vibrotactile stimulation using a bone vibrator and then a PTA and speech audiometry was performed using a GSI61 audiometer. An immittance test was carried out using GSI Tympstar Pro. Tympanometry using a $226 \mathrm{~Hz}$ probe tone was carried out and reflexometry was obtained bilaterally to ipsilateral and contralateral stimulation. Distortion product otoacoustic emissions (DPOAEs) were recorded bilaterally using a ILO V6 instrument with a frequency range of $1,000 \mathrm{~Hz}$ to $8,000 \mathrm{~Hz}$. Based on basic audiological findings, the test battery was further extended to include auditory brainstem response (ABR) tracked using clicks, $500 \mathrm{~Hz}$ and $4,000 \mathrm{~Hz}$ tone burst stimuli. Late latency response (LLR) was also performed using a $1000 \mathrm{~Hz}$ tone burst stimulus at an intensity of $70 \mathrm{dBnHL}$. Both the auditory evoked potential testing was done using Biologic Navigator Pro Version 7.2. Detailed speech and language evaluation was carried out. The child was referred for an imaging test; however, they could not follow-up due to financial constraint and travel inconvenience and thus is a limitation.

The child was very well conditioned to vibrotactile 
stimulation but was completely unresponsive to puretone audiometry. The PTA revealed no response to air conduction stimulation even at maximum limits from $250 \mathrm{~Hz}$ to $8,000 \mathrm{~Hz}$ in both ears (Figure 1). Similarly, there was no response to bone conduction stimulation of the right ear from $250 \mathrm{~Hz}$ to $4,000 \mathrm{~Hz}$. She could understand gestural commands and it could be understood that she was not able to hear any sounds. The

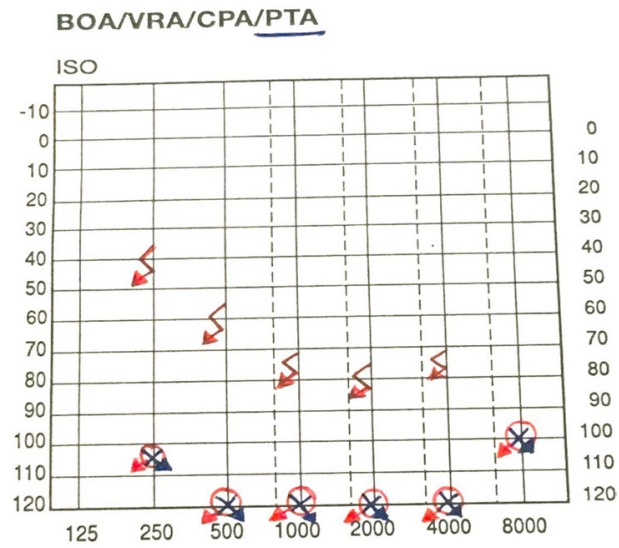

Figure 1. Pure tone audiometry (PTA) showing bilateral profound hearing loss. middle ear analysis was done using tympanometry, which revealed normal middle ear functioning with bilateral 'A' type tympanograms (Figure 2). A peculiar finding was noted in reflexometry. Both ears showed absent acoustic reflexes on ipsilateral recording, but acoustic reflexes were present in the right ear on contralateral presentation of the stimulus and absent in the left ear. The consistency of this result was confirmed by repeating three trials. This made us extend our test battery. DPAOEs revealed normal emissions with SNR (signal to noise ratio) more than $6 \mathrm{dBSPL}$ from $1.4 \mathrm{KHz}-8 \mathrm{KHz}$ with good amplitude bilaterally as shown in Figure 3. Subsequently, bilateral peak I, III \&V of ABR (Figure 4) could be recorded until $30 \mathrm{dBnHL}$ in response to clicks and peak $\mathrm{V}$ till $30 \mathrm{dBnHL}$ in response to $4,000 \mathrm{~Hz}$ tone burst stimuli. Peak $\mathrm{V}$ was present till $40 \mathrm{dBnHL}$ and absent at $30 \mathrm{dBnHL}$ in response to $500 \mathrm{~Hz}$ tone burst stimuli, bilaterally. This clearly suggested that the child had normal peripheral hearing and thus brought us to the next level of testing.

The possible reason for her unresponsiveness to sounds could lie in the central auditory system. Since she had no response to PTA, no other behavioral test would help us look into the central auditory system. This led us to the selection of LLR, which revealed that there was absence of all the components of LLR i.e.
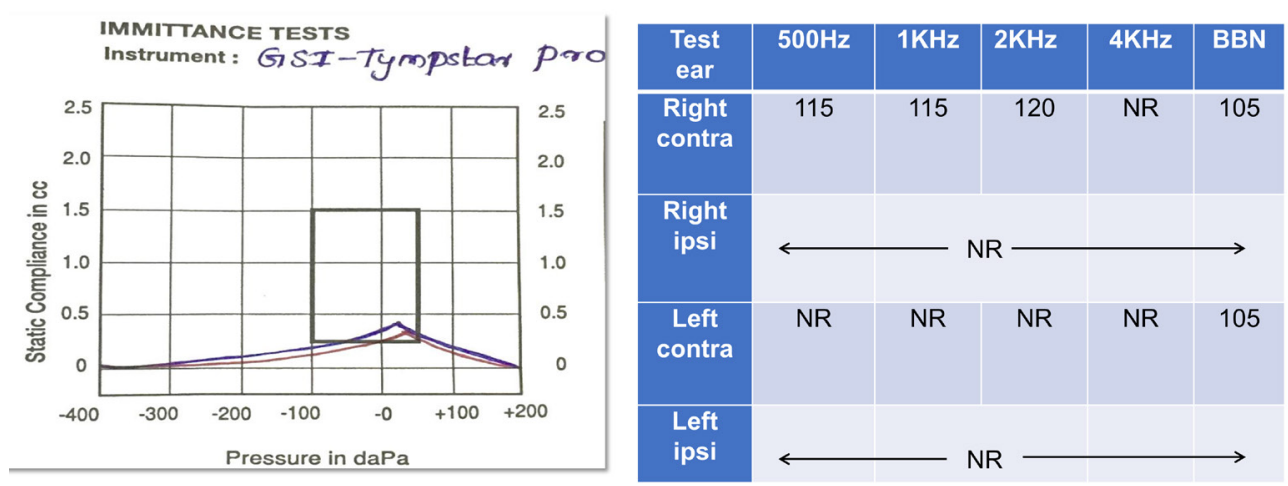

Figure 2: Immittance showing bilateral 'A' type of tympanogram with contralateral acoustic reflexes present at higher intensities.

RIGHT EAR

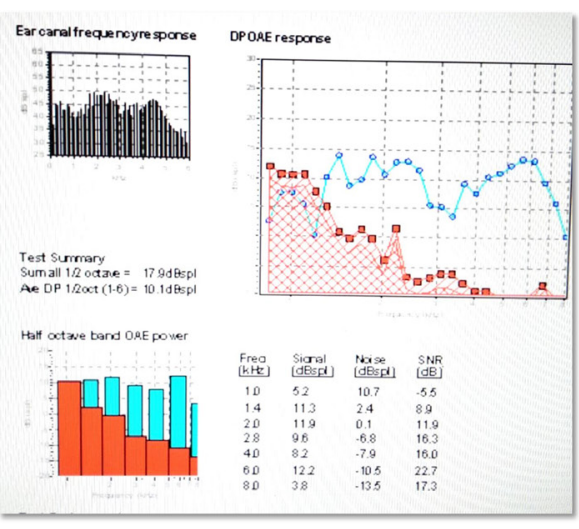

LEFT EAR

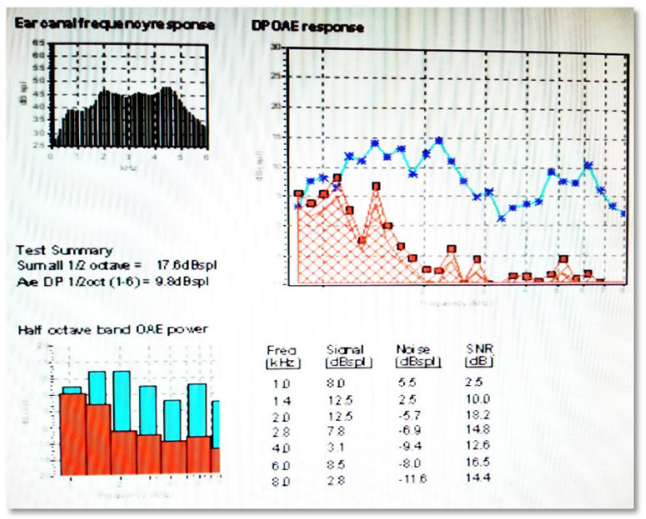

Figure 3. DPgram showing bilateral presence of distortion product otoacoustic emissions (DPOAEs). 
RIGHT EAR

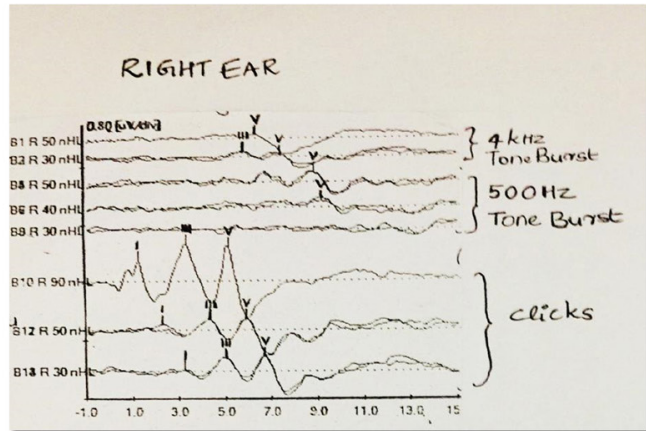

LEFT EAR

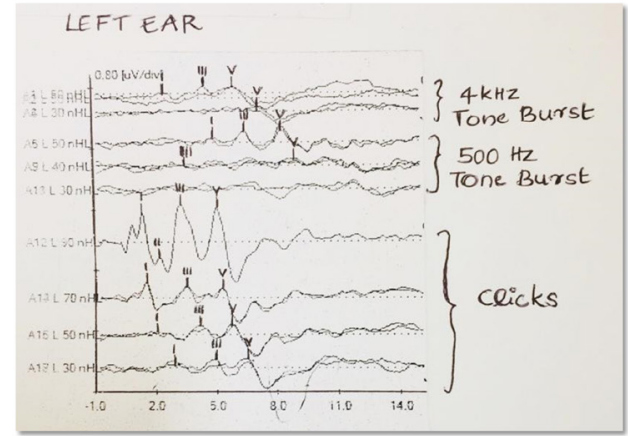

Figure 4. Auditory brainstem response (ABR) showing bilateral presence of waves I, III \& V in response to clicks, $500 \mathrm{~Hz} \& 4 \mathrm{KHz}$ toneburst stimuli.

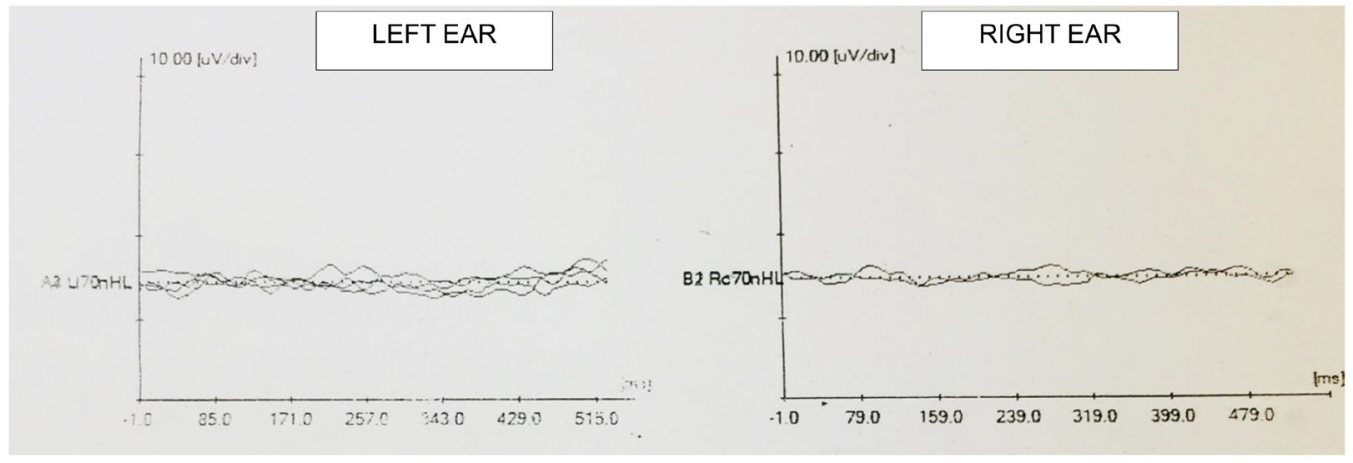

Figure 5. Late latency response (LLR) showing bilateral absence of peaks.

P1, N1, P2 \& N2 at 70dBnHL. Two recordings ensured the replicability of each wave and both recordings showed no replicable peaks throughout the time window bilaterally. Figure 5 shows absent LLR bilaterally on two recordings of each ear. Detailed speech \& language evaluation reveals delay in receptive and expressive language skills (receptive and expressive language age of 0-0.5 months respectively, based on Assessment of Language Development test). Reading and writing could not be assessed, as the child was not sent to school. The child was recommended for magnetic resonance imaging (MRI) scan but did not follow up.

\section{Discussion}

It is known that cortical deafness is an extremely rare condition (4) reported in the literature with single case studies. Among them, meningitis as a cause for cortical deafness is even rarer. With the advent of cochlear implantation, meningitis has caught the attention of several researchers and clinicians as it presents with peripheral hearing loss and cochlear ossification (11) thereby needing immediate management. In certain cases, meningitis also presents with normal peripheral hearing but cortical deafness has not been reported so far in the recent literature. Hood et al. (3) in their discussion have quoted a case of cortical deafness due to meningitis (9). The symptoms of the child presented in this study were very much similar to those of pre-lingual deafness with totally no response to pure-tone audiometry or to any speech stimulus presented at the maximum levels of the audiometer. Most often, the studies on cortical deafness reveal complete deafness on puretone audiometry results, although cases with residual hearing or even normal hearing cannot be ruled out (4). There can also be instances of inconsistent or variable responses to sound (12).

The bilateral absence of acoustic reflexes to ipsilateral stimulation and the presence of reflexes in only one ear to contralateral stimulation at very high intensities can be explained with a possible lesion in the reflex pathway. However, this is contrary to the general findings of cortical lesion where acoustic reflexes are present at normal levels (3). In addition, the ABR is also normal in both the ears with good morphology and lower thresholds suggesting normal brainstem function. Among the objective tests performed on patients with cortical deafness ABR has found to be normal in many cases $(4,12)$ as they have normal peripheral hearing. However, the possibility of a lesion in the reflex pathway could not be explained. The presence of normal peripheral mechanism has been further highlighted in this case with the presence of normal outer hair cell function as evident from DPOAEs. Authors $(4,13)$ also report normal otoacoustic emissions (OAEs) in a cortical lesion. The only objective test that explained the absence of response 
to pure-tone audiometry was the absence of cortical response, LLR. The diagnosis of cortical deafness is based predominantly on the objective measures (3) with middle latency response (MLR) and LLR being sensitive. In this study, however the MLR was not done on this case. The upper brainstem and subcortical structures could have been explored using MLR (14) and possibly a correlation with reflexometry would have given a better understanding of the condition.

This child would need to rely on sign language or an alternative augmentative communication to meet her communication demands. Early intervention in children with cortical deafness would have improved the quality of life to some extent. In cases of cortical deafness, post-stroke patients are recommended for follow up for the possibility of long-term recovery (4). In India, the awareness towards, the importance of follow up is lacking. This case had never sought an audiological evaluation until a free hearing screening was set up. The study also highlights the outreach to the rural population.

A test battery in the diagnostic protocol of meningitis must include a combination of subjective and objective tests. Even if the ABR exhibits normal results, the incorporation of LLR is a mandate. Since the focus of tests in a patient with meningitis has always remained on identifying ossification of the cochlea, many cases of cortical deafness would have been overlooked. The study also highlights the importance of contralateral acoustic reflexes which would give an insight into the involvement of the brainstem not picked up by the ABR and suggests how sometimes physiological tests might be more informative than electrophysiologcal tests. The availability of imaging would have been ideal for correlating the present audiological results but is not present because the client failed to follow up and this remains the drawback of this paper.

A combination of both audiological and imaging tests are recommended in order to investigate cortical deafness (4). However, cortical deafness can be identified accurately by means of an audiological test battery, which turns out to be economical especially for patients from a rural background.

\section{Acknowledgements}

Authors wish to thank Dr. Madhuri Gore for her valuable insights during the case study.

\section{References}

1. Landau WM, Goldstein R, Kleffner FR. Congenital aphasia: a clinicopathologic study. Neurology. 1960;
10:915-921.

2. Jerger J, Weikers NJ, Sharbrough FW 3rd, Jerger S. Bilateral lesions of the temporal lobe. Acta Otolaryngol Supp1. 1969; 258:1-51.

3. Hood LJ, Berlin CI, Allen P. Cortical deafness: a longitudinal study. J Am Acad Audiol. 1994; 5:330-342.

4. Brody RM, Nicholas BD, Wolf MJ, Marcinkevich PB, Artz GJ. Cortical deafness: a case report and review of the literature. Otol Neurotol. 2013; 34:1226-1229.

5. Musiek FE, Charette L, Morse D, Baran JA. Central deafness associated with a midbrain lesion. J Am Acad Audiol. 2004; 15:133-151; quiz 172-173.

6. Zaidan E, Baran JA. Gaps-in-noise (GINC) test results in children with and without reading disabilities and phonological processing deficits. Int J Audiol. 2013; 52:113-123.

7. Michel F, Peronnet F, Schott B. A case of cortical deafness: clinical and electrophysiological data. Brain Lang. 1980; 10:367-377.

8. Narayanan S, Majeed KA, Subramaniam G, Narayanan A, Navaf KM. A case of cortical deafness due to bilateral heschl gyrus infarct. Case Rep Med. 2017; 2017:6816748.

9. Lechevalier B, Rossa Y, Eustache F, Schupp C, Boner L, Bazin C. Case of cortical deafness sparing the music area. Rev Neurol (Paris). 1984; 140:190-201. (in French).

10. Axon PR, Temple RH, Saeed SR, Ramsden RT. Cochlear ossification after meningitis. Am J Otol. 1998; 19:724729.

11. Durisin M, Büchner A, Lesinski-Schiedat A, Bartling S, Warnecke A, Lenarz T. Cochlear implantation in children with bacterial meningitic deafness: the influence of the degree of ossification and obliteration on impedance and charge of the implant. Cochlear Implants Int. 2015; 16:147-158

12. Musiek FE, Baran JA, Shinn JB, Guenette L, Zaidan E, Weihing J. Central deafness: an audiological case study. Int J Audiol. 2007; 46:433-441.

13. Kaga K, Shinjo Y, Enomoto C, Shindo M. A case of cortical deafness and loss of vestibular and somatosensory sensations caused by cerebrovascular lesions in bilateral primary auditory cortices, auditory radiations, and postcentral gyruses - complete loss of hearing despite normal DPOAE and ABR. Acta Otolaryngol. 2015; 135:389-394.

14. Baran JA, Bothfeldt RW, Musiek FE. Central auditory deficits associated with compromise of the primary auditory cortex. J Am Acad Audiol. 2004; 15:106-116.

Received June 26, 2020; Revised July 29, 2020; Accepted August 20, 2020

*Address correspondence to:

Deena Priya, Department of Hearing Studies, Dr. S.R. Chandrasekhar Institute of Speech and Hearing, Hennur main road, Lingarajpuram, Bengaluru- 560084, Karnataka, India.

E-mail: deenapriya91@gmail.com

Released online in J-STAGE as advance publication September 4, 2020. 